\title{
Random distributed feedback fiber laser of ultimate efficiency
}

\author{
I. D. Vatnik ${ }^{1}$, D. V. Churkin ${ }^{1,2,3}$, E. V. Podivilov ${ }^{1,3}$, S. A. Babin ${ }^{1,3}$ \\ ${ }^{1}$ Institute of Automation and Electrometry SB RAS, Novosibirsk 630090, Russia \\ ${ }^{2}$ Aston Institute of Photonic Technologies, Aston University, Birmingham, B4 7ET, UK \\ ${ }^{3}$ Novosibirsk State University, Novosibirsk 630090, Russia \\ novolaser@gmail.com
}

\begin{abstract}
We demonstrate a random fiber laser of ultimate efficiency. More than 2 Watts are generated from $0.5 \mathrm{~W}$ of pump excess over the generation threshold. At higher power, an optical efficiency corresponds to the quantum limit.
\end{abstract}

OCIS codes: (140.3510) Lasers, Raman; (190.5650) Raman effect

\section{Introduction}

Since its first demonstration [1], random fiber lasers based on distributed feedback (DFB) provided by Rayleigh scattering (RS) and distributed gain provided by stimulated Raman scattering (SRS) have proven to possess a lot of attractive features [2]. Recently is has been pointed out that that the total efficiency of a random DFB fiber laser with co-directional pumping has exponential dependence on fiber length that is defined by linear attenuation of pump and laser light in the fiber [3-4]. So, decreasing of the cavity length may sufficiently enhance the laser efficiency, but the threshold pump power will be increased too. Finding a balance between these factors will result in maximum efficiency at moderate pumping. Here we design a short random DFB fiber laser and demonstrate the ultimate power performances and efficiency.

\section{Experiment and discussion}

To design the high-efficiency random DFB fiber laser, we use a forward-pumped random fiber laser configuration [5]. The laser is based on a short span $(\mathrm{L}=850 \mathrm{~m})$ of a phosphosilicate fiber featured by a large Stokes shift $\left(1330 \mathrm{~cm}^{-1}\right)$ of $\mathrm{P}_{2} \mathrm{O}_{5}$-related Raman gain peak [6]. As a pump laser, we use an $\mathrm{Yb}$-doped fiber laser (YDFL) emitting at $1115 \mathrm{~nm}$. The output end of the fiber is spliced to an angle-polished connector to avoid Fresnel reflection at the fiber end facet. We have inspected the output connector reflection by means of optical time domain reflectometer and estimated the parasitic reflection to be of $10^{-6}$ that two order of magnitude lower than the integral Rayleigh backscattering strength $R_{R S} \sim 10^{-4}$. The opposite end (i.e. $1.3 \mu \mathrm{m}$ port of WDM) was terminated by a Sagnac fiber-loop mirror that allows one to reduce the generation threshold by a factor of 2 as compared with the random DFB fiber laser configuration without mirror [2].

The laser has a generation threshold $P_{\text {pump }}^{t h}=5.5 \mathrm{~W}$, Fig.1a. Above the threshold, the residual pump power at the laser output is decreased down to zero value, whereas the generated Stokes wave power at $1308 \mathrm{~nm}$ reaches the value of $P_{s}^{\text {out }}=7.3 \mathrm{~W}$ at the input pump power $P_{p}^{\text {in }}=11 \mathrm{~W}$. To prove the results, we have performed numeric simulation based on power balance model described in [2,7], and found it to be in good agreement with experiment.

In the short random DFB fiber laser we observe the exceptional dependence of the output power on the pump power. First of all, the generation power increases rapidly above the threshold reaching the output power value of 2 $\mathrm{W}$ at only $0.5 \mathrm{~W}$ excess of the pump power over the threshold (i.e. at $P_{p}^{i n}=6 \mathrm{~W}$ ), Fig. 1a. The differential generation efficiency defined as $d P_{s}^{o u t} / d P_{p}^{\text {in }}$ demonstrates the corresponding behaviour: just above the threshold it amounts to several hundred percent and then gradually falls down being stabilized at the level of $\sim 75 \%$ for pump powers of $\geq 10 \mathrm{~W}$, see Fig. 1b, Note that in conventional lasers the output power dependence is usually close to linear with a small region of the exponential growth around the generation threshold because of the ASE influence [6].

The second important fact is the ultimate absolute and quantum generation efficiencies of the demonstrated random DFB fiber laser high above the generation threshold. Indeed, the absolute optical efficiency defined as the ratio of the output generation power to the input pump power, $\eta=P_{s}^{\text {out }} / P_{p}^{\text {in }}$, approaches $66 \%$, see Fig. $1 \mathrm{c}$. Here the output power depends linearly on the input pump power with almost the same slope as the output pump in absence of laser generation (compare the dashed curve and dots on Fig. 1a). This, in particular, means that the energy is transferred from one spectral band (1115 nm, pump wave) to the new spectral band (1308 nm, Stokes wave) in the most efficient way. 

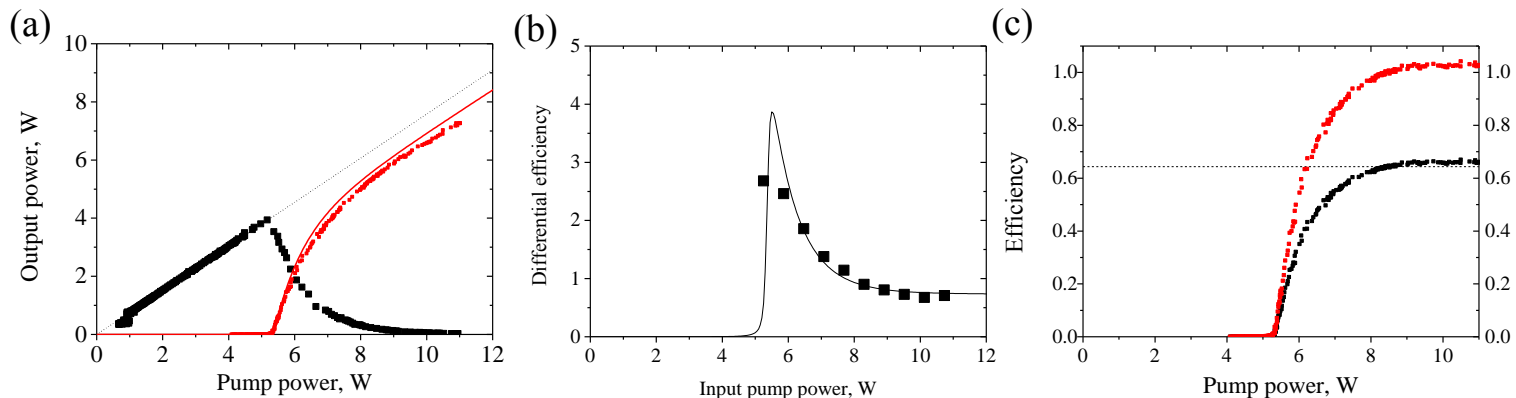

Fig.1. (a) The output power at $1308 \mathrm{~nm}$ (red points) together with the transmitted pump power at $1115 \mathrm{~nm}$ (black points). The dashed black line is the transmitted output pump power under assumption of gain absence. Red line is from numerics. (b) Slope efficiency from the experiment (squares) and numeric (line) (c) Black squares - total optical efficiency as the ratio of the output generation power to the input pump power. The horizontal dashed line is the maximum conversion efficiency $\eta_{\max }=\exp \left(-\alpha_{p} L\right) h v_{s} / h v_{p}=64 \%$. Red squares - the quantum conversion efficiency defined as the ratio of the generated photon number at the laser output to the pump photon number at the system output if the Raman conversion is absent.

To highlight this fact, we calculate the quantum efficiency by taking into account the linear losses for the pump wave, $P_{p}^{\text {out }} / P_{p}^{\text {in }}=\exp \left(-\alpha_{p} L\right) \approx 0.76$, and the quantum limit of energy conversion, $h v_{s} / h v_{p}=0.85$. Here $v_{s}$ and $v_{p}$ are Stokes and pump light frequencies correspondingly, $\alpha_{p}=0.331 / \mathrm{km}$ is linear attenuation at the pump frequency. The short random RDF fiber laser demonstrates efficiency slightly higher than the maximum possible conversion efficiency, $\eta_{\max }=\exp \left(-\alpha_{p} L\right) h v_{s} / h v_{p}=64 \%$, see Fig. 1c.

And what is more important, corresponding quantum conversion efficiency can reach and even exceed $100 \%$ in our case. We calculate the number of the photons emitted from the random laser output Stokes as $N_{s}^{\text {out }}=P_{s}^{\text {out }} / h v_{s}$. At the same time, the number of pump photons reaching the far end of the fiber span in a passive regime (if there is no generation of the Stokes wave) is given by $N_{p}^{o u t}=P_{p}^{i n} e^{-\alpha_{p} L} / h v_{p}$. We plot the ratio of the generated Stokes photons to the pump photons at the fiber output $(z=L)$, and found that the energy transfer to a new spectral band in the short random fiber laser is accompanied with the increase of the photon number by several percent: $N_{s}^{\text {out }} /$ $N_{p}^{\text {out }}=1.03$, corresponding rise is clearly seen in Fig. 1c.

To conclude, we design a short random DFB fiber laser of an ultimate power performance. At high power over the generation threshold, the random fiber laser has an ultimate quantum efficiency $(\sim 100 \%)$ of the photons conversion rate to a different spectral band: each absorbed pump photon is converted to the emitted laser photon. Moreover, the conversion efficiency defined for the output radiation reaches $103 \%$. This effect is attributed to the specific distribution of the number of photons over the random fiber laser's length and sufficiently lower linear losses for the Stokes photons compared to that for the pump photons. At that, the obtained absolute optical efficiency of $66 \%$ corresponds to maximum possible value defined by quantum limit and attenuation of pump and laser waves

\section{References}

1. S. K. Turitsyn, S. A. Babin, A. E. El-Taher, P. Harper, D. V. Churkin, S. I. Kablukov, J. D. Ania-Castanon, V. Karalekas, and E. V. Podivilov, "Random distributed feedback fibre laser," Nat. Photonics 4, 231-235 (2010).

2. S. K. Turitsyn, S. A. Babin, D. V. Churkin, I. D. Vatnik, M. Nikulin, and E. V. Podivilov, "Random distributed feedback fibre lasers," Phys. Rep. DOI:10.1016/j.physrep.2014.02.011 (2014).

3. S. A. Babin, A. E. El-Taher, P. Harper, E. V. Podivilov, and S. K. Turitsyn, "Tunable random fiber laser," Phys. Rev. A 84, 021805 (2011).

4. D.V. Churkin, A.E. El-Taher, I.D. Vatnik, J.D. Ania-Castañón, P. Harper, E.V. Podivilov, S.A. Babin, S.K. Turitsyn, "Experimental and theoretical study of longitudinal power distribution in a random DFB fiber laser", Optics Express 20, 11178-11188 (2012)

5. D. V. Churkin, S. A. Babin, A. E. El-Taher, P. Harper, S. I. Kablukov, V. Karalekas, J. D. Ania-Castañón, E. V. Podivilov, and S. K. Turitsyn, "Raman fiber lasers with a random distributed feedback based on Rayleigh scattering," Phys. Rev. A 82, 033828 (2010).

6. I. A. Bufetov, M. M. Bubnov, Y. V. Larionov, O. I. Medvedkov, S. A. Vasiliev, M. A. Melkoumov, A. A. Rybaltovsky, S. L. Semjonov, E. M. Dianov, V. F. Khopin, F. Durr, H. G. Limberger, and M. Zeller, "Highly efficient one-and two-cascade Raman lasers based on phosphosilicate fibers," Laser Phys 13, 234-239 (2003).

7. I. D. Vatnik, D. V. Churkin, and S. A. Babin, "Power optimization of random distributed feedback fiber lasers," Opt. Express 20, 28033 (2012). 